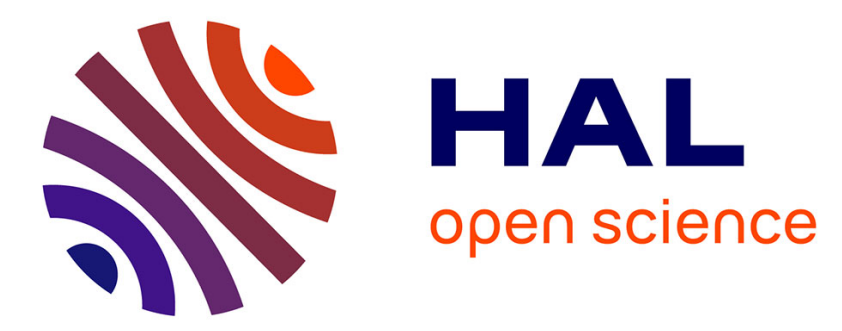

\title{
Adsorption and Desorption of a Phospholipid from Single Microbubbles under Pulsed Ultrasound Irradiation for Ultrasound-Triggered Drug Delivery
}

Makiko Nakata, Nozomi Tanimura, Daisuke Koyama, Marie Pierre Krafft

\section{- To cite this version:}

Makiko Nakata, Nozomi Tanimura, Daisuke Koyama, Marie Pierre Krafft. Adsorption and Desorption of a Phospholipid from Single Microbubbles under Pulsed Ultrasound Irradiation for UltrasoundTriggered Drug Delivery. Langmuir, 2019, 10.1021/acs.langmuir.8b03621 . hal-03018465

\section{HAL Id: hal-03018465 https://hal.science/hal-03018465}

Submitted on 22 Nov 2020

HAL is a multi-disciplinary open access archive for the deposit and dissemination of scientific research documents, whether they are published or not. The documents may come from teaching and research institutions in France or abroad, or from public or private research centers.
L'archive ouverte pluridisciplinaire HAL, est destinée au dépôt et à la diffusion de documents scientifiques de niveau recherche, publiés ou non, émanant des établissements d'enseignement et de recherche français ou étrangers, des laboratoires publics ou privés. 


\section{Adsorption and Desorption of a Phospholipid from Single Microbubbles under Pulsed Ultrasound Irradiation for Ultrasound-Triggered Drug Delivery}

Makiko Nakata1, Nozomi Tanimura1, Daisuke Koyama2*, Marie Pierre Krafft3

1Faculty of Life and Medical Sciences, Doshisha University, 1-3 Tataramiyakodani, Kyotanabe, Kyoto,610-0321, Japan

2Faculty of Science and Engineering, Doshisha University, 1-3 Tataramiyakodani, Kyotanabe, Kyoto 610-0321, Japan

3Institut Charles Sadron (CNRS), University of Strasbourg, 23 rue du Loess, 67034 Strasbourg, France *dkoyama@mail.doshisha.ac.jp

Abstract: Microbubbles have potential for applications as drug and gene delivery systems in which the release of a substance is triggered by an ultrasonic pulse. In this paper, we discuss the adsorption and desorption of a film of phospholipid on the surface of a single microbubble under ultrasound irradiation. Our optical observation system consisted of a high-speed camera, a laser Doppler vibrometer, and an ultrasound cell; 1,2-dimyristoyl-sn-glycero-3-phosphocholine (DMPC) was used as the surfactant. The adsorption of the DMPC molecules onto the surface of the bubble was evaluated by measuring the contact angle between the bubble and a glass plate. A decrease of the contact angle of the bubble indicates desorption of the DMPC molecules from the bubble surface into the surrounding aqueous solution. The amount of DMPC molecules adsorbed on the bubble's surface is shown to decrease over time after bubble generation. The type and intensity of the pulsed ultrasound waves were varied so as mimic ultrasound triggered drug release. Increasing the number of cycles and the amplitude of the sound pressure of the pulsed ultrasound yielded a greater increase of the contact angle. We also measured the radial vibrations of the microbubbles in the ultrasound field. The vibrational characteristics of the microbubbles and the desorption characteristics of the DMPC molecules showed the same variation; namely, a greater sound pressure amplitude induced greater vibrational displacement and a larger amount of molecular desorption under resonance conditions. These results support the possibility of controlling drug release with pulsed ultrasound in a microbubble-based drug delivery system.

\section{Introduction}

Developments in ultrasound techniques have produced remarkable noninvasive and simple to use ultrasound imaging techniques, which enable the internal state of living bodies to be observed in real time [1-6]. Ultrasound techniques are widely used in medical diagnosis, and research is developing in the field of therapeutic techniques [7-10]. In ultrasound imaging techniques, microbubbles are injected into blood vessels and used as contrast agents to enhance image contrast. These microbubbles consist 
of an internal gas and a surrounding molecular film such as a lipid shell [11,12]. The internal gas is usually saturated with a volatile fluorocarbon, which acts as a poorly water-soluble osmotic agent and co-surfactant, thus improving the bubble stability and enabling control over the microbubble behavior $[13,14]$. The behavior of microbubbles under ultrasound irradiation is a basic characteristic of ultrasound imaging methods in which signals reflected from the microbubbles are analyzed [15-17]. When microbubbles are exposed to low amplitude sound pressure fields the bubbles spherical vibrations synchronize with the pressure change, and the vibrational mode of the microbubbles depends on the sound pressure [18]. If the pressure amplitude increases, the vibrational mode shifts to a non-spherical mode with surface waves propagating on the bubble's surface. A further increase of the pressure amplitude induces intense vibrations, resulting in collapse of the microbubbles, accompanied by microjets of internal gas that penetrate the shell because the shape of the bubbles cannot be maintained [19]. Microbubbles are expected to have applications as drug delivery systems (DDSs) [20-26]. However, intravascular administration of bubbles carries a risk of side effects. To reduce this risk it is desirable that the bubbles be targeted to tissues, as by antigen-antibody reactions, or that the release of the drug cargo be triggered at specific sites. In DDSs that use ultrasound, the adsorption of microbubbles to the target tissues is assisted by Bjerknes forces induced by ultrasound. Drug or gene release to the cells can be achieved by triggering bubble collapse, which is accompanied by generation of microjets and shock waves [27]. However, van Wamel et al. reported that drug transfer was accelerated even when the sound pressure amplitude was comparatively small [28], implying that generation of microjets or shockwaves may not be required for drug release. Therefore, to control transfer of the drug, the vibration and collapse characteristics under ultrasound irradiation of microbubbles with a self-assembled molecular shell need to be clarified to achieve microbubbles suitable for clinical use. Hence, we used 1,2-dimyristoyl-sn-glycero-3-phosphocholine (DMPC) as the shell-forming surfactant to define the ultra-sound trigger pulses required for safe implementation of this drug delivery procedure.

There have been numerous reports on bubble vibration and collapse characteristics based on both experimental [29-32] and theoretical [33-35] investigations. We have reported on the adsorption characteristics of the poloxamer Pluronic F-68 on air- or $F$-hexane-saturated bubbles and on the effects of a film of this water-soluble block copolymer on bubble vibration under ultrasound irradiation [11]. For the ultrasound-assisted DDS, the mechanism of molecular desorption from the bubble surface should be clarified to control the amount of drug release. In this paper, we focus on desorption of DMPC molecules from a bubble triggered by pulsed ultrasound and the effects of ultrasound on the bubble vibration behavior.

\section{Materials and Methods}

In the case of sub-millimeter-sized microbubbles shelled with phospholipids, molecular adsorption 
to the gas-liquid interface can be evaluated by bubble profile analysis tensiometry [36]. Here, we investigated the adsorption and desorption characteristics of DMPC from single bubbles, tens of micrometers in size, using an instrument based on an ultrasound transducer and a high-speed camera. The phospholipid, 1,2- dimyristoyl-sn-glycero-3-phosphocholine (DMPC), was purchased from NOF Corporation [CAS Registry No. 18194-24-6; $M_{\mathrm{w}}$ value of 677.9] and used as a bubble shell-forming material. A 198-mg portion of DMPC was added to $250 \mathrm{~mL}$ of phosphate buffered saline (Fujifilm Wako Pure Chemical Corporation, Osaka, Japan). In consideration of applications in clinical research, we added $132 \mathrm{mg}$ of polyethylene glycol monostearate $\left[\mathrm{CH}_{3}\left(\mathrm{CH}_{2}\right)_{16} \mathrm{COO}\left(\mathrm{CH}_{2} \mathrm{CH}_{2} \mathrm{O}\right)_{2} \mathrm{H}\right.$; Fujifilm Wako Pure Chemical Corporation, Osaka, Japan; CAS Registry No. 106-11-6; $M_{\mathrm{w}}$ value of 372.66] to the solution because the surface of DMPC bubbles can be modified through the polyethylene glycol $[37,38]$.

Figure 1 shows the optical observational system. A bolt-clamped Langevin-type ultrasound piezoelectric transducer with a resonance frequency of $38.8 \mathrm{kHz}$ (Fuji Ceramics, Fujinomiya, Japan) was fitted to the bottom of a rectangular transparent cell $(75 \times 75 \times 60 \mathrm{~mm} 3)$. By exciting the transducer with an electrical sinusoidal signal at a resonance frequency of $38.8 \mathrm{kHz}$, a half-wavelength longitudinal vibration mode was generated in the length direction of the transducer and the sound wave radiated toward the aqueous phase through the bottom of the cell. The cell was filled with the phospholipid solution and the level of the liquid was controlled so that an acoustic standing wave was generated in the vertical direction by multireflections of the sound wave. Air microbubbles were generated in the observational cell by electrolysis $[39,40]$. The size distribution of the air microbubbles is shown in Figure 2. The DC voltage of $6 \mathrm{~V}$ was applied to a copper wire for electrolysis by a DC power supply (DK-801, Sunhayato, Tokyo, Japan). Air bubbles with a radius ranging from 20 to 150 $\mu \mathrm{m}$ were generated and the average radius was approximately $80 \mu \mathrm{m}$. The size distribution of microbubbles can be controlled by the electric voltage and control of the electric voltage caused the bubble to attach to the surface of a glass plate fixed at the center of the cell. The bubbles attached to the glass plate were positioned in the antinodal plane of the sound pressure in the acoustic standing wave to excite the bubble efficiently when a continuous sinusoidal signal was applied to the ultrasound transducer. Because the thickness of the glass plate was much smaller than the wavelength of the standing wave, the plate did not disturb the acoustic field. After the microbubbles were attached to the glass plate, the surfactant molecules in the solution began to adsorb to the surface of the bubbles and formed a self-assembled monomolecular film around the bubbles $[41,42]$

The microbubbles attached on the glass plate were observed from the horizontal direction by a highspeed camera (HPV-1, Shimadzu, Kyoto, Japan) equipped with a long-distance microscope. Continuous light from a xenon lamp was directed toward the observational cell and received by the camera. The contact angle of the microbubble with the glass plate was measured from the captured image. This measurement allowed the adsorption of the phospholipid molecules to the bubble surface 
to be evaluated from changes in the interfacial tension between air, solution, and the glass. A photograph of a microbubble on the glass plate is shown in Figure 3(a). The image of the bubble appears dark owing to refraction of the backlight on the bubble surface. The contact angle of the bubble $\theta$ was calculated geometrically as [43]:

$$
\theta=\cos ^{-1}\left(\frac{2 H}{D}\right),
$$

where $H$ is the distance between the center of the bubble and the surface of the glass plate and $D$ is the diameter of the bubble in the horizontal direction. The change in contact angle was measured at 2-min intervals with the camera. Approximately $30 \mathrm{~min}$ after their generation, the microbubbles were subjected to a single round of pulsed ultrasound irradiation. The number of cycles of pulsed ultrasound was then changed from 10 to 500 cycles and the sound pressure amplitude from 1.8 to $20 \mathrm{kPa}$. The sizes of microbubbles used in the experiments (20 to $150 \mu \mathrm{m}$ ) are comparatively larger than that of ultrasound contrast agents for clinical use ( 1 to $5 \mu \mathrm{m})$ since the image resolution of the camera is 1.69 $\mu \mathrm{m} /$ pixel.

When the microbubbles were exposed to the pulsed waves, radial vibrations were generated on the bubble's surface (gas-liquid interface). The sensor head of a laser Doppler vibrometer (LDV, NLV2500, PI Polytech, Waldbronn, Germany) with an objective lens (M Plan Apo 20× or M Plan Apo 100×, Mitutoyo, Kanagawa, Japan) was set above the observation cell, allowing measurement of the bubble vibrations in the standing wave. The laser beam of the LDV was focused perpendicularly to the top of the bubble to measure the radial component of the vibration through the Doppler effect of the reflected light, and the initial bubble size was determined simultaneously using the CCD camera installed in the sensor head; the image resolution was $1.02 \mu \mathrm{m} /$ pixel. The focal spot size of the LDV beam was $1.5 \mu \mathrm{m}$, which is relatively small compared with the bubble size (tens of micrometers) used in this experiment. All the experiments were conducted at $22^{\circ} \mathrm{C}$. In total, 159 bubble samples of different sizes were measured.

\section{Results and Discussion}

We investigated the adsorption characteristics of DMPC molecules on the bubble's surface. After an air microbubble was generated in solution, the contact angle of the bubble changed gradually over time because the DMPC molecules in solution began to reach the surface of the bubble and the surface tension of the bubble decreased. In the case of an air bubble adhering to a glass plate, the contact angle $\theta$ decreases as the surface tension decreases, and the adsorption kinetics of the DMPC molecules can be characterized by $\theta$. The variation of the surface tension depends on the experimental conditions, such as the concentration of the phospholipid and the composition of the internal gas [11]. Considering the time required to reach saturation of the DMPC adsorption at the bubble surface, pulsed ultrasound was irradiated $1800 \mathrm{~s}$ after the generation of the microbubbles, and the change in the contact angle 
was measured continuously.

Representative photographs of adhering microbubbles before $(t=30 \mathrm{~min})$ and after ultrasonication $(t=31 \mathrm{~min})$ are shown in Figures 3(a) and (b), respectively. The contact angle of the bubble clearly increased under the influence of the pulsed ultrasound. We determined a relationship between pulse duration of the ultrasound and the change in the contact angle of the bubble. The changes in the contact angle for several microbubbles with radii ranging from 20 to $150 \mu \mathrm{m}$, excited with a sound pressure amplitude of $7.3 \mathrm{kPa}$ at $38.8 \mathrm{kHz}$ are shown in Figure 4. In all cases, the contact angle decreased exponentially with time ( $t=0$ to $30 \mathrm{~min}$ ) and increased significantly after ultrasound irradiation (at $t$ $=30 \mathrm{~min}$ ). A greater number of cycles of pulsed ultrasound resulted in a greater increase in the contact angle. After the transitory increase induced by the ultrasound irradiation, the contact angle decreased gradually over time ( $t=30 \mathrm{~min}$ to $47 \mathrm{~min}$ ). Notably, the initial contact angles of the samples at $t=0 \mathrm{~s}$ were different and ranged from 20 to 40 degrees because time was needed to control the focal point of the camera on the bubble samples after bubble generation by electrolysis; thus, " $t=0 \mathrm{~s}$ " does not correspond to a specific moment after bubble generation and there are different time lags between bubble generation and the start of observations. The uncertainty in the measurement of the contact angle is attributed mainly to the resolution of the optical images $(1.69 \mathrm{um} / \mathrm{pixel})$ and to the fact that the position of the air-liquid interface was determined from the change in brightness of the captured images; the maximum error was approximately 6 degrees. These results imply that the DMPC molecules on the surface of the bubble momentarily desorbed under ultrasound irradiation and that the molecules in the solution readsorbed to the bubble surface over time. The relationship between the number of cycles of pulsed ultrasound and the increase in the contact angle when irradiated at $t=30$ min is shown in Figure 5. The plots and error bars indicate the average values and standard deviations for five samples having bubble sizes ranging from 20 to $150 \mu \mathrm{m}$ although the dependence of the microbubble behavior on bubble size is discussed later. The change in the contact angle increased with the number of cycles of pulsed ultrasound and saturated at approximately 100 cycles. This result indicates that there is a threshold of vibrational acceleration on the bubble surface for the desorption of DMPC molecules if the forces acting on the molecules at the bubble surface are assumed to be proportional to the vibrational acceleration. A decrease in the contact angle was observed by the highspeed camera (shutter speed of 4000 frames/s), immediately after ultrasound irradiation, indicating that the time constant of the molecular desorption was extremely short and can be estimated to $2 \mathrm{~ms}$ at most.

We also investigated the DMPC adsorption and desorption characteristics by changing the sound pressure amplitude of the pulsed ultrasound. Figure 6 shows the changes in the contact angle of a range of microbubbles caused by ultrasound pulsed at 50 cycles at $38.8 \mathrm{kHz}$. Similar to the results presented in Fig. 4, the pulsed ultrasound was radiated at $t=30 \mathrm{~min}$, resulting in transitory increases of the contact angle owing to desorption of DMPC molecules. The contact angle increased when the sound 
pressure amplitude increased (Figure 7). Unfortunately, further increases of the sound pressure amplitude above $20 \mathrm{kPa}$ induced violent vibrations of the microbubbles, which caused their detachment from the glass plate.

We measured the vibrational characteristics of the DMPC microbubbles to investigate the relationship between the molecular desorption of DMPC from the bubble's surface and the bubble vibrations. The behavior of microbubbles under the influence of ultrasound depends on several parameters, including bubble size, sound pressure amplitude, and ultrasound frequency [43]. The vibrational characteristics of the DMPC bubbles having a radius from 20 to $140 \mu \mathrm{m}$ under ultrasound irradiation are shown in Figure 8. An acoustic standing wave was generated in the cell under a continuous ultrasound wave at $38.8 \mathrm{kHz}$. We measured the bubble vibration after $30 \mathrm{~min}$ and the time required for saturation of the bubble surface by DMPC. The horizontal and vertical axes indicate the initial bubble radius $R 0$ without ultrasound irradiation and the vibrational displacement amplitude of the bubble normalized by the initial radius $(\Delta R / R 0)$ under ultrasound irradiation, respectively, for several sound pressure amplitudes. These resonance curves of the DMPC bubbles indicate that the resonance radius at $38.8 \mathrm{kHz}$ is approximately $60 \mu \mathrm{m}$ and that greater sound pressure amplitudes generate greater vibrational displacement amplitudes. The experimental results in Fig. 5, indicate that the increase in contact angle plateaued at approximately 100 cycles of ultrasound. This effect is related to the transient response of the ultrasound transducer and the bubble vibrations because the sound pressure amplitude generated by the transducer and the vibrational amplitude of microbubbles attained a steady state after a transient state during which they increased gradually. Waveforms of ultrasound pulsed at 50 cycles measured with a needle hydrophone and the transient vibrations of a bubble having a radius of $80 \mu \mathrm{m}$ are shown in Figure 9. The bubble vibration reached a steady state after approximately 25 cycles. Therefore, tens of cycles of ultrasound are required to achieve steady state bubble vibration and induce desorption of the DMPC molecules. Additionally, the asymptotic value of the increase of the contact angle was approximately 10 degrees, which suggests that either some of the DMPC molecules remained adsorbed to the bubble surface or that molecular adsorption and desorption on the bubble's surface occur simultaneously under the ultrasound irradiation.

Bubble vibration under ultrasound irradiation is essentially a nonlinear phenomenon. When the sound pressure amplitude is comparatively small (less than $10 \mathrm{kPa}$ ), the bubble generally behaves linearly and exhibits spherical vibration. If the sound pressure amplitude increases, the vibrational amplitude also increases and the vibrational mode becomes nonlinear and non-spherical. Further increase in sound pressure induces bubble collapse. Therefore, bubbles that have reached their resonance size show larger vibration amplitudes when subjected to a greater sound pressure, which will induce greater desorption of DMPC molecules. We can estimate the amount of molecular desorption from the vibration characteristics of the microbubbles. The results in Fig. 7 show the same variation as the vibrational characteristics shown in Fig. 8; i.e., a greater sound pressure induced greater molecular 
desorption owing to a higher vibrational amplitude of the bubbles.

\section{Conclusions}

We investigate and discuss the formation of a self-assembled DMPC film on a bubble's surface under pulsed ultrasound irradiation. The molecular adsorption and desorption of the phospholipid were evaluated by measuring the contact angle of single microbubbles with a high-speed camera. The contact angle increased when a pulsed ultrasound wave was applied, which implies that the DMPC molecules desorbed from the bubble's surface. This effect by ultrasonication was not limited in the DMPC solution and the same phenomenon was observed in the case with other surfactants such as Pluronic $\mathrm{F}_{68}$ [11]. The contact angle increased with the number of cycles of pulsed ultrasound applied and plateaued at approximately 100 cycles. The contact angle also increased when the sound pressure amplitude of the ultrasound was increased. We measured the vibrational characteristics of the microbubbles in order to investigate the relationship between bubble vibrations and molecular desorption. The changes in vibrational amplitude and molecular desorption showed the same variation, i.e., both behaviors increased together with the sound pressure amplitude, implying that the amount of drug released from bubbles can be controlled by the shape of the pulsed ultrasound in a drug delivery system. Although, in this paper, we have examined only the deposition from a self-assembled monomolecular film, we intend to conduct the experiments using other surfactants that form a multilayer film and micelles.

\section{Acknowledgement}

This work was partially supported by the Heiwa Nakajima Foundation. We thank Andrew Jackson, $\mathrm{PhD}$, from Edanz Group (www.edanzediting.com/ac) for editing a draft of this manuscript.

\section{References}

[1] Fan, C.; Caleap, M.; Pan, M.; Drinkwater, B. W. A comparison between ultrasonic array beamforming and super resolution imaging algorithms for non-destructive evaluation. Ultrasonics 2014, 54, 1842-1850.

[2] Rawool, N. M.; Forsberg, F.; Winder, A. A.; Hume, E. Power Doppler assessment of vascular changes during fracture treatment with low-intensity ultrasound. J. Ultrasound Med. 2003, 22, $145-153$.

[3] Ge, N.; Sun, S. Endoscopic ultrasound: An all in one technique vibrates virtually around the whole internal medical field. J. Trans. Intern. Med. 2014, 2,104-106.

[4] Gennisson, J-L.; Deffieux, T.; Fink, M.; Tanter M, Ultrasound elastography: Principles and techniques. Diagn. Interv. Imaging. 2013, 94, 487-495.

[5] Sarvazyan, P. S.; Urban, M. W.; Greenleaf, J. F., Acoustic waves in medical imaging and 
diagnostics. Ultrasound Med. Biol. 2013, 39, 1133-1146.

[6] Sun, J-T.; Huang, C-Y.; Huang, Y-S.; Sim, S-S.; Chong, K-M.; Wang, H-P.; Lien, W-C. Prehospital ultrasound. J. Med. Ultrasound 2014, 22, 71-77.

[7] Treat, L. H.; Vykhoodtseva, N.; Zhang, Y.; Hynynen, K. Target delivery of doxorubicin to the rat brain at therapeutic levels using MRI-guided focused ultrasound. Int. J. Cancer 2007, 121, 901-907.

[8] Chen, Y.; Liu, L. Modern methods for delivery of drugs across the blood-brain barrier. Adv. Drug Deliver. Rev. 2012, 64, 640-665.

[9] Wood, A. K. W.; Sehgal, C. M. A review of low-intensity ultrasound for cancer therapy. Ultrasound Med. Biol. 2015, 41, 905-928.

[10] Leong-Poi, H.; Kuliszewski, M. A.; Lekas, M.; Sibbald, M.; Teichert-Kuliszewska, K.; Klibanov, A. L.; Stewart, D. J.; Lindner J. R. Therapeutic arteriogenesis by ultrasound-mediated VEGF165 plasmid gene delivery to chronically ischemic skeletal muscle. Circ. Res. 2007, 101, 295-303.

[11] Ando, Y.; Tabata, H.; Sanchez, M.; Cagna, A.; Koyama, D.; Krafft, M. P. Microbubbles with a selfassembled poloxamer shell and a fluorocarbon inner gas. Langmuir 2016, 32, 12461-12467.

[12] Lee, S.; Kim, D. H.; Needham, D. Equilibrium and dynamic interfacial tension measurements at microscopic interfaces using a micropipet technique. 2. Dynamics of phospholipid monolayer formation and equilibrium tensions at the water-air interface. Langmuir, 2001, 17, 5544-5550.

[13] Szijjarto, C.; Rossi, S.; Waton, G.; Krafft, M. P. Effects of perfluorocarbon gases on the size and stability characteristics of phospholipid-coated microbubbles - Osmotic effect versus interfacial film stabilization. Langmuir 2012, 28, 1182-1189.

[14] Krafft, M. P. Fluorine in medical microbubbles - Methodologies implemented for engineering and investigating fluorocarbon-based microbubbles, J. Fluorine Chem. 2015, 177, 19-28.

[15] Gennisson, J-L.; Deffieux, T.; Fink, M.; Tanter, M. Ultrasound elastography: Principles and techniques. Diagn. Interv. Imaging 2013, 94, 487-495.

[16] Coli, S.; Magnoni, M.; Sangiorgi, G.; Marrocco-Trischitta, M. M.; Melisurgo, G.; Mauriello, A.; Spagnoli, L.; Chiesa, R.; Cianflone, D.; Maseri, A. Contrast-enhanced ultrasound imaging of intraplaque neovascularization in carotid arteries. J. Am. Coll. Cardiol. 2008, 52, 223-230.

[17] Sebag, F.; Vaillant-Lombard, J.; Berbis, J.; Griset, V.; Henry, J-F.; Petit, P.; Oliver, C. Shear wave elastography: A new ultrasound imaging mode for the differential diagnosis of benign and malignant thyroid nodules. J. Clin. Endocrinol. Metab. 2010, 95, 5281-5288.

[18] Chu, P.; Pax, R.; Li, R.; Langlois, R.; James, A. F. Using sound to study the effect of frothers on the breakaway of air bubbles at an underwater capillary. Langmuir 2017, 33, 3200-3207.

[19] de Jong, N.; Emmer, M.; van Wamel, A.; Versluis, M. Ultrasonic characterization of ultrasound contrast agents. Med. Biol. Eng. Comput. 2009, 47, 861-873.

[20] Miller, D; Smith, N; Bailey, M.; Czarnota, G.; Hynynen, K.; I. Makin; Bioeffects Committee of 
the American Institute of Ultrasound in Medicine. Overview of therapeutic ultrasound applications and safety considerations. J. Ultrasound Med. 2012, 31, 623-634.

[21] Liu, H-L.; Hua, M-Y.; Yang, H-W.; Huang, C-Y.; Chu, P-C.; Wu, J-S.; Tseng, I-C.; Wang, J-J.; Yen, T.-C.; Chen, P.-Y.; Wei, K-C. Magnetic resonance monitoring of focused ultrasound/magnetic nanoparticle targeting delivery of therapeutic agents to the brain. Proc. Natl. Acad. Sci. USA 2010, 107, 15205-15210.

[22] Dijkmans, P. A.; Juffermans, L. J.; Musters, R. J.; van Wamel, A.; ten Cate F. J.; van Gilst, W.; Visser, C. A.; de Jong, N.; Kamp, O. Microbubbles and ultrasound: from diagnosis to therapy. Eur. J. Echocardiogr. 2004, 5, 245-246.

[23] Cho, K.; Wang, X.; Nile, S.; Chen, Z. G.; Shin, D. M. Therapeutic nanoparticles for drug delivery in cancer. Clin. Cancer Res. 2008, 14, 1310-1316.

[24] Lin, C-H.; Chen, C-H.; Lin, Z-C.; Fang J-Y. Recent advances in oral delivery of drugs and bioactive natural products using solid lipid nanoparticles as the carriers. J. Food Drug Anal. 2017, 25, 219- 234.

[25] Kang, S-N.; Hong, S-S.; Kim, S-Y.; Heungchan, O.; Lee, M-K.; Lim, S-J. Enhancement of liposomal stability and cellular drug uptake by incorporating tributyrin into celecoxib-loaded liposomes. Asian J. Pharm. Sci. 2013, 8, 128-133.

[26] Bariya, S. H.; Mukesh, C. G.; Mehta, T. A.; Sharma O. P.; Microneedles: An emerging transdermal drug delivery system. J. Pharm. Pharmacol. 2012, 64, 11-29.

[27] Doinikov, A. A. Bjerknes forces and translational bubble dynamics, Bubble and particle dynamics in acoustic fields: Modern trends and applications. ISBN. 2005, 81-7736-284-4.

[28] van Wamel, A.; Kooiman, K.; Harteveld, M.; Emmer, M.; ten Gate, F. J.; Versluis, M.; de Jong, N. Vibrating microbubble poking individual cells: Drug transfer into cells via sonoporation. $J$. Control. Release 2006, 112, 149-155.

[29] Zhao, S.; Kruse, D. E.; Ferrara, K. W.; Dayton, P. A. Acoustic response from adherent targeted contrast agents. J. Acoust. Soc. Am. 2006, 120, EL63-EL69.

[30] de Jong, N.; Emmer, M.; Chin, C. T.; Bouakaz, A.; Mastik, F.; Lohse, D.; Versluis, M. "Compression-only" behavior of phospholipid-coated contrast bubbles. Ultrasound Med. Biol. 2007, 33, 653-656.

[31] Koyama, D.; Osaki, A.; Kiyan, W.; Watanabe, Y. Acoustic destruction of a micro-capsule having a hard plastic shell. IEEE Trans. Ultrason., Ferroelect., Freq. Contr. 2006, 53, 1314-1321.

[32] Koyama, D.; Kotera, H.; Kitazawa, N.; Yoshida, K.; Nakamura, K.; Watanabe, Y.; Vibration of a single microcapsule with a hard plastic shell in an acoustic standing wave field. IEEE Trans. Ultrason., Ferroelect., Freq. Contr. 2011, 58, 737-743.

[33] Church, C. C. The effects of an elastic solid surface layer on the radial pulsations of gas bubbles. J. Acoust. Soc. Am. 1995, 97, 1510-1521. 
[34] Chatterjee, D.; Sarkar, K. A Newtonian rheological model for the interface of microbubble contrast agents. Ultrasound Med. Biol. 2003, 29, 1749-1757.

[35] Marmottant, P.; van der Meer, S.; Emmer, M.; Versluis, M.; de Jong, N.; Hilgenfeldt, S.; Lohse, D. A model for large amplitude oscillations of coated bubbles accounting for buckling and rupture. J. Acoust. Soc. Am. 2005, 118, 3499-3505.

[36] Nguyen, P. N.; Waton, G.; Vandamme, T.; Krafft, M. P.; Behavior of an adsorbed phospholipid monolayer submitted to prolonged periodical surface density variations, Angew. Chem. Int. Ed. 2013, 52, 6404-6408.

[37] Hagisawa, K.; Nishioka, T.; Suzuki, R.; Takizawa, T.; Maruyama, K.; Takase, B.; Ishihara, M.; Kurita, A.; Yoshimoto, N.; Ohsuzu, F.; Kikuchi, M. Enhancement of ultrasonic thrombus imaging using novel liposomal bubbles targeting activated platelet glycoprotein IIb/IIIa complex-in vitro and in vivo study. Int. J. Cardiol. 2011, 152, 202-206.

[38] Yokoi, Y.; Yoshida, K.; Otsuki, Y.; Watanabe, Y. Area density of streptavidin can be evaluated by the number density of biotinylated microbubbles, Appl. Phys. Lett. 2017, 110, 073702.

[39] Kothandaraman, A.; Harker, A.; Ventikos, Y.; Edirisinghe, M. Novel preparation of monodisperse microbubbles by integrating oscillating electric fields with microfluidics. Micromachines 2018, 9, 497.

[40] van der Linde, P.; Moreno Soto, Á. M.; Peñ as-Lopez, P.; Rodríguez-Rodríguez, J.; Lohse, D.; Gardeniers, H.; van der Meer, D.; Fernandez Rivas, D. Electrolysis-driven and pressurecontrolled diffusive growth of successive bubbles on microstructured surfaces. Langmuir 2017, $33,12873-12886$.

[41] Nicolas, J. A.; Lynn, M. W.; Shelley, L. A. A microtensiometer to probe the effect of radius of curvature on surfactant transport to a spherical interface. Langmuir 2010, 26, 13310-13319.

[42] Kobayashi, M.; Terayama, Y.; Yamaguchi, H.; Terada, M.; Murakami, D.; Ishihara, K. Wettability and antifouling behavior on the surfaces of superhydrophilic polymer brushes. Langmuir 2012, 28, 7212-7222.

[43] Yoshida, K.; Morioka, S.; Kagawa, Y.; Koyama, D.; Watanabe, Y. Power-law dependence describing subharmonic generation from a non-spherically oscillating bubble. Acoust. Sci. \& Tech. 2015, 36, 191-200. 


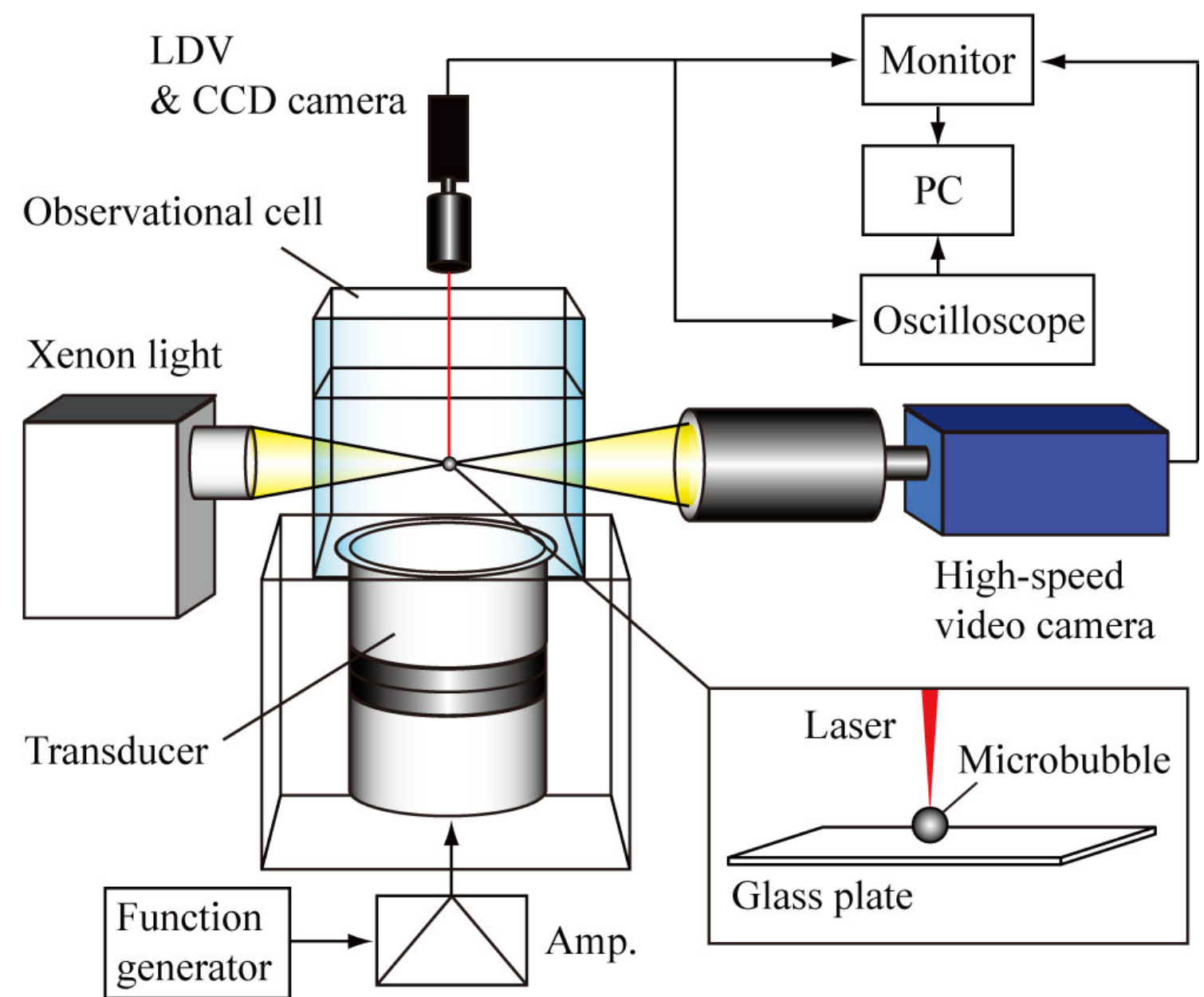

Figure 1. Observational setup for measuring the adsorption characteristics of DMPC molecules onto a single microbubble. 


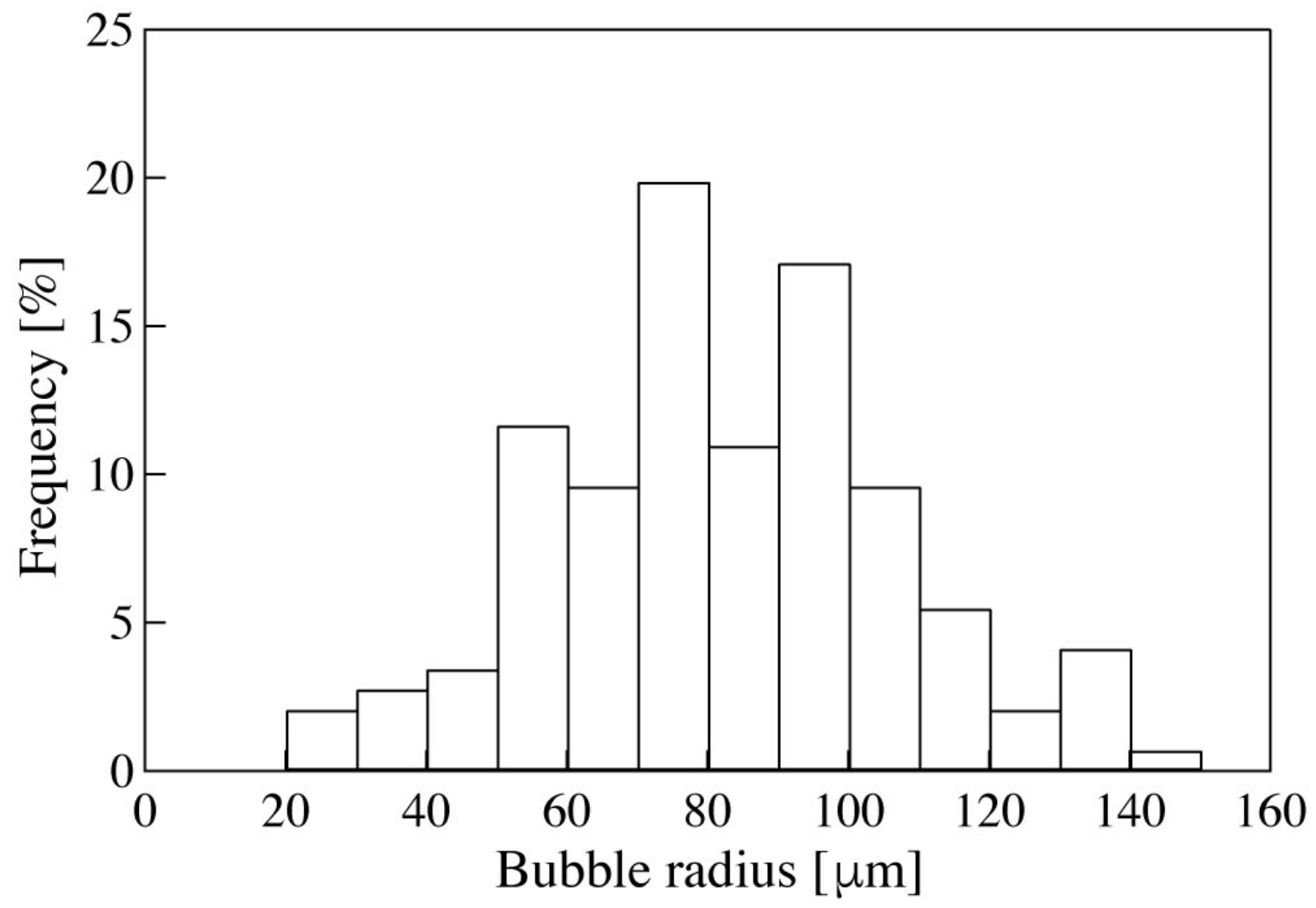

Figure 2. Size distribution of microbubbles generated by electrolysis. 

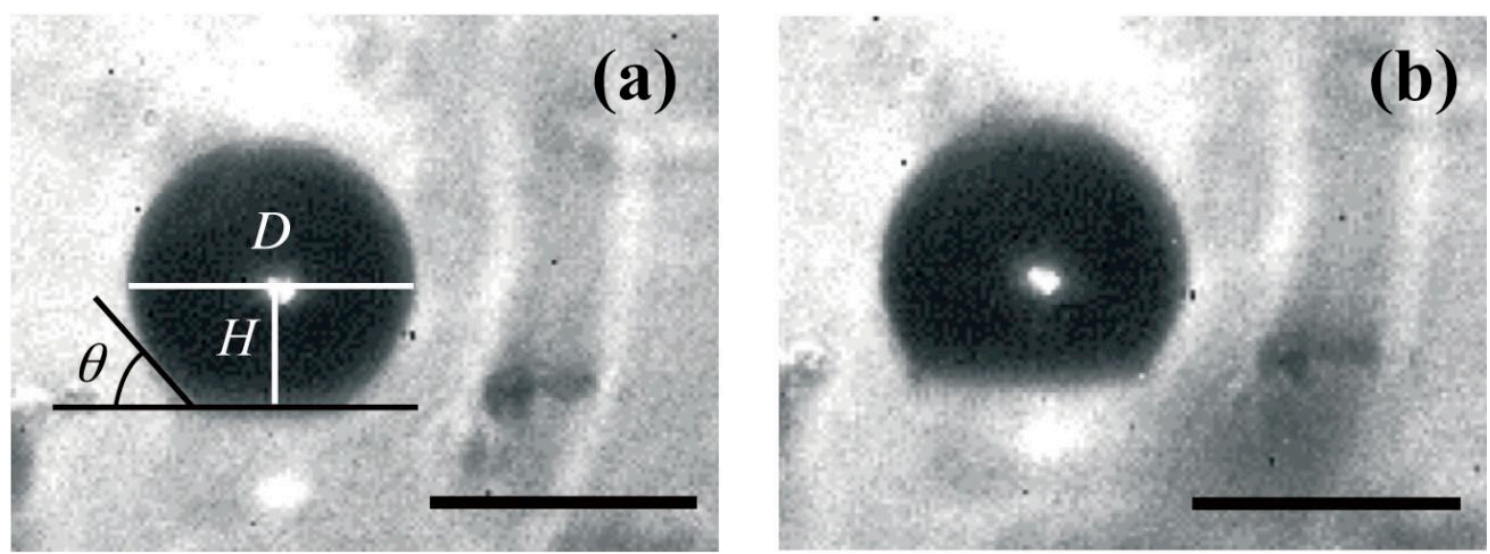

Figure 3. Images of microbubbles adhered to the glass plate with (a) small ( $t=30 \mathrm{~min})$ and (b) large contact angles $(t=31 \mathrm{~min}) . D$ and $H$ indicate the diameter of the bubble and the distance between the center of the bubble and the surface of the glass plate. Scale bars corresponds to $100 \mathrm{~m}$. 

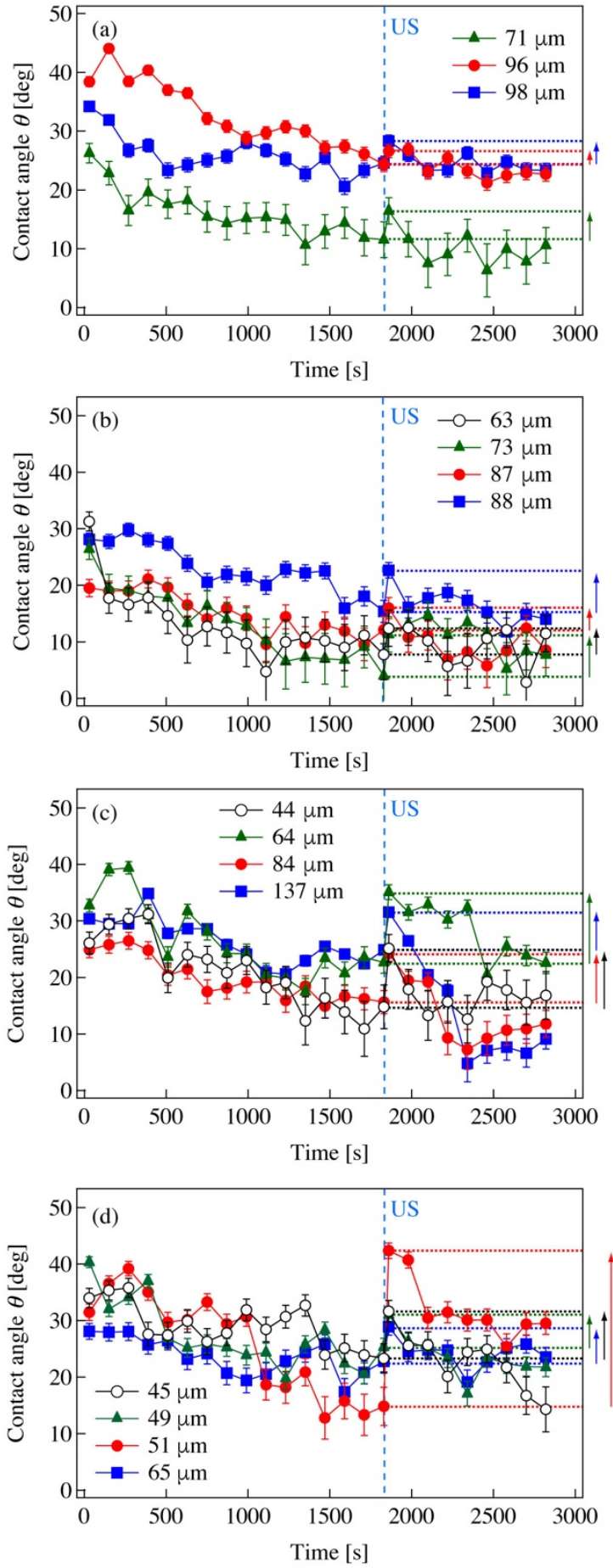

Figure 4. Changes in contact angle for several microbubbles subjected to (a) 10, (b) 30, (c) 50, and (d) 100 cycles of pulsed ultrasound at a sound pressure amplitude of $7.3 \mathrm{kPa}$ at $38.8 \mathrm{kHz}$. Vertical dotted lines at $t=30 \mathrm{~min}$ indicate the onset of ultrasound irradiation. Horizontal dotted lines and arrows indicate the transitory increase in the contact angle induced by ultrasonication. Error bars indicate the uncertainty of the image resolution. 


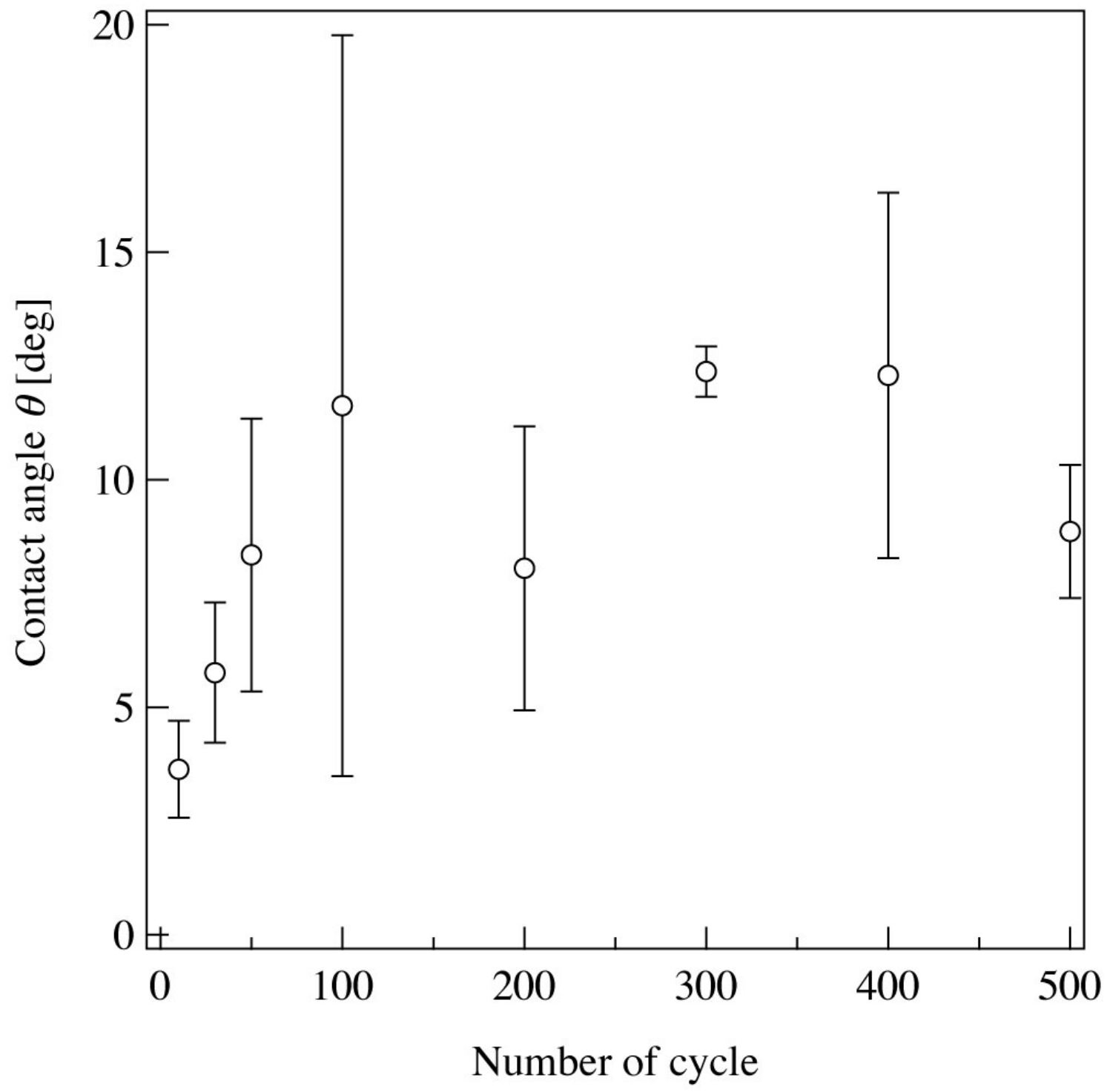

Figure 5. Relationship between the number of cycles of pulsed ultrasound and the increase of the contact angle of the microbubbles at $t=30 \mathrm{~min}$. 

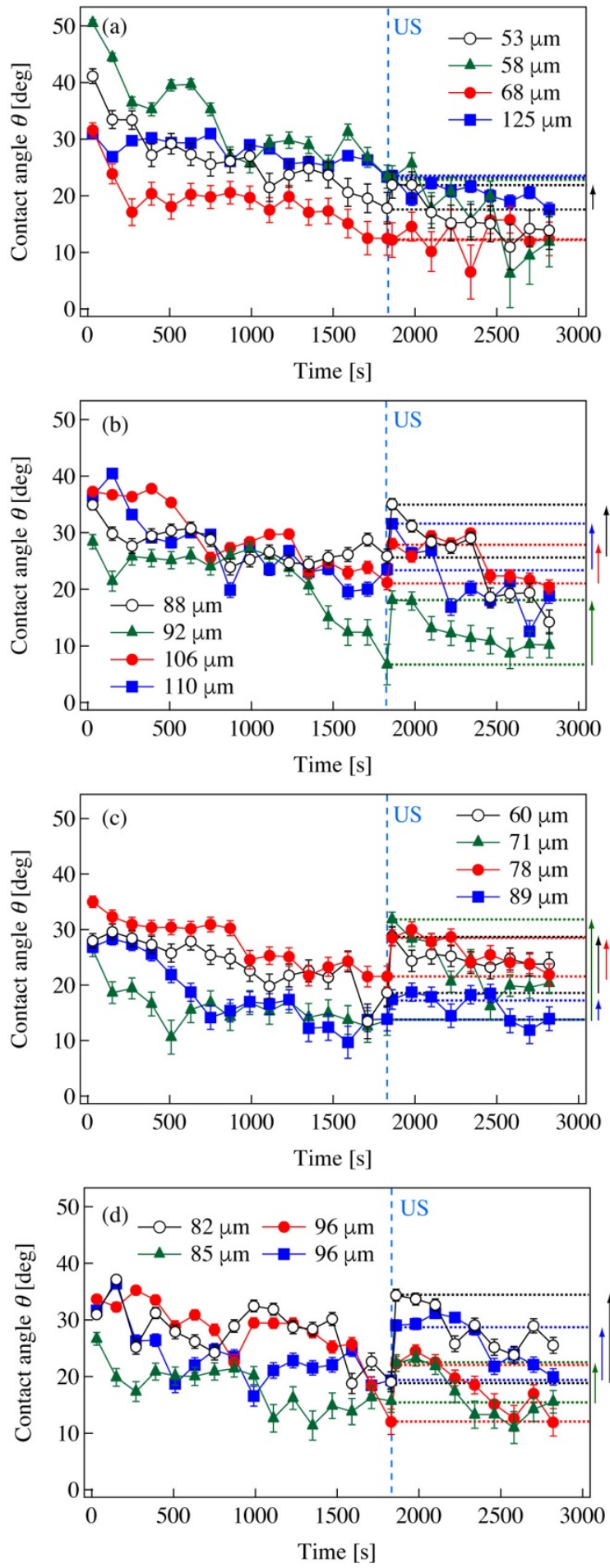

Figure 6. Changes in the contact angle for several microbubbles subjected to 50 cycles of pulsed ultrasound with sound pressure amplitudes of (a) 1.8, (b) 10, (c) 15, and (d) $20 \mathrm{kPa}$ at $38.8 \mathrm{kHz}$. Vertical dotted lines at $t=30 \mathrm{~min}$ indicate the onset of the ultrasound irradiation. Horizontal dotted lines and arrows indicate the transitory increase in the contact angle induced by ultrasonication. 


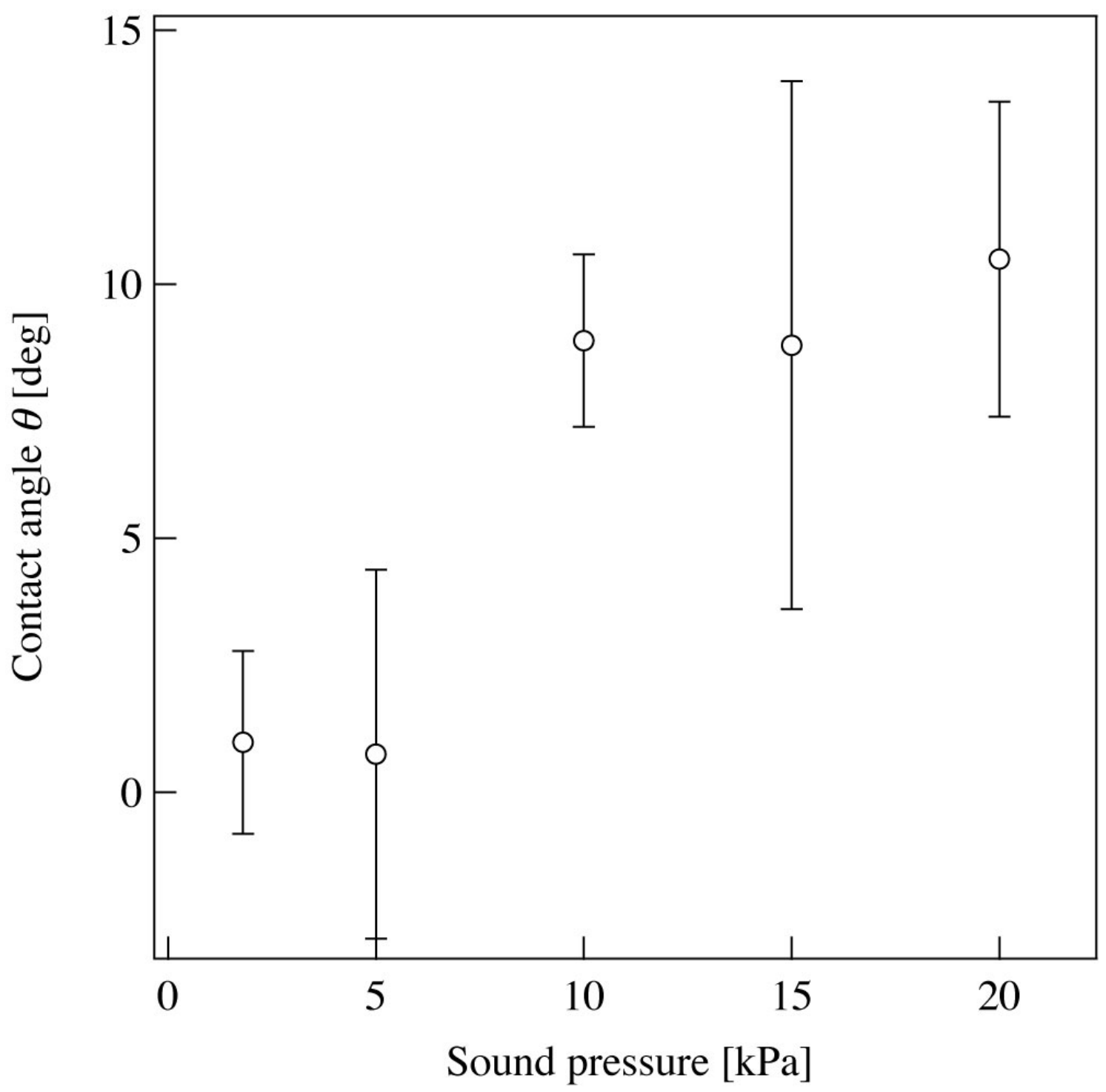

Figure 7. Relationship between the sound pressure amplitude of the pulsed ultrasound and the increase in contact angle of the microbubbles from $t=30 \mathrm{~min}$ (onset of pulsed ultrasound irradiation) to 31 $\min$. 


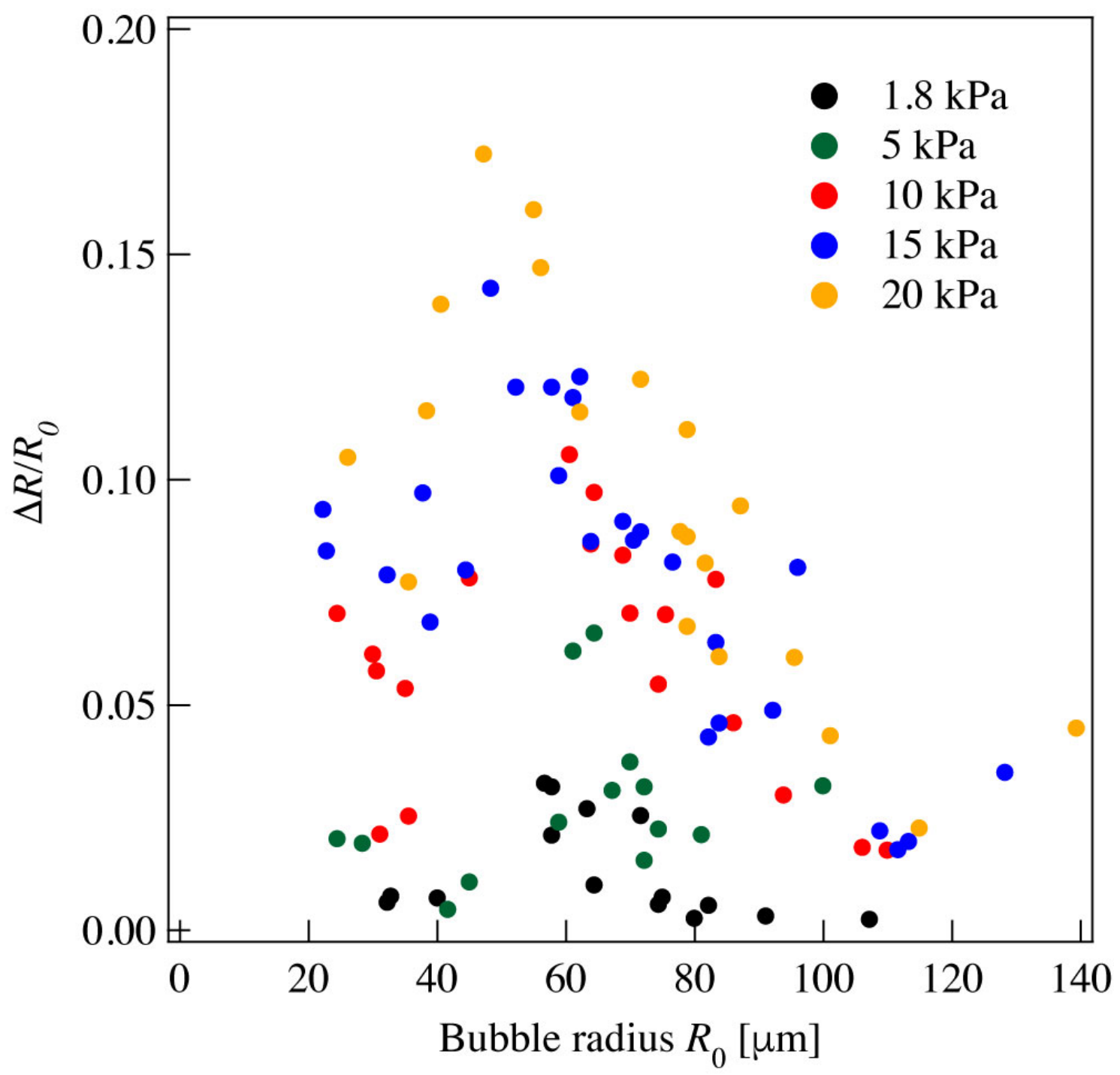

Figure 8. Relationship between initial bubble radius and normalized displacement amplitude for single microbubbles excited by an ultrasound standing wave at $38.8 \mathrm{kHz}$ with several sound pressure amplitudes after $t=30 \mathrm{~min}$. 

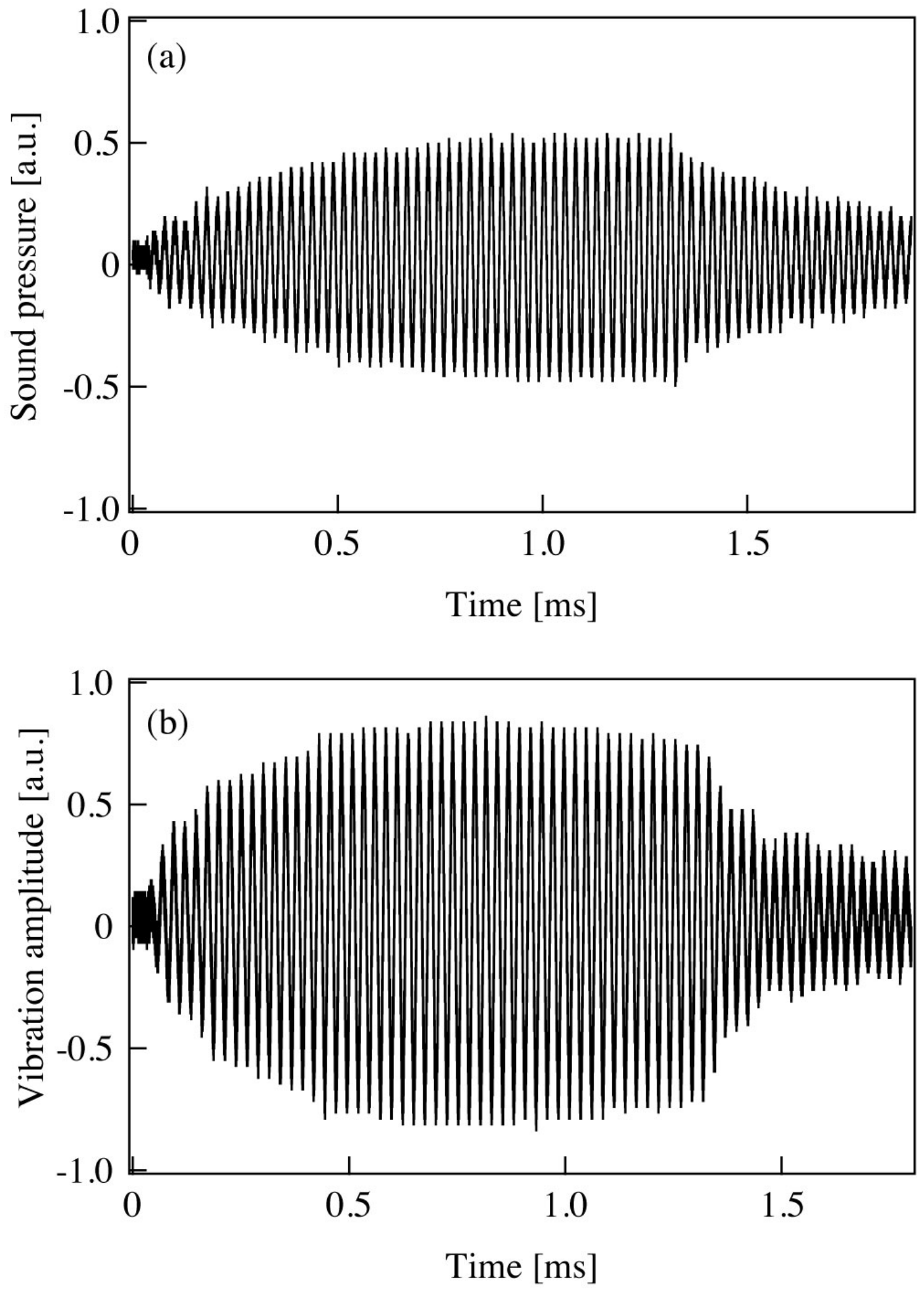

Figure 9. Waveforms of (a) pulsed ultrasound of 50 cycles at a frequency of $38.8 \mathrm{kHz}$ measured by a needle hydrophone and (b) transient vibration of a bubble having a radius of 80 m as measured by 
the LDV 